\title{
Recognising the need for innovation in digitalising urgent or advance care planning in adults aged 70 years and over following emergency admission
}

\author{
Authors: Anna-Maria Bielinska, ${ }^{1}$ Adetokunbo Obanobi, ${ }^{2}$ Julia Riley $^{3}$ and Catherine Urch ${ }^{4}$
}

\begin{abstract}
Aims
Approximately one in three hospital inpatients may be in their last year of life in Scotland; ${ }^{1}$ however, no data exist for England, therefore this study aimed to establish the 1-year mortality of a cohort of patients aged 70 years and above admitted to a large London hospital trust, to explore the need for innovation in urgent or advance care planning and sharing electronic care plans.
\end{abstract}

\section{Methods}

Admission data were obtained via Cerner, the electronic patient record, over 6 months from 1 May to 31 October 2014 at Imperial College Healthcare NHS Trust. Presentations were stratified according to emergency overnight admission and age. Follow-up data on mortality over 1 year were analysed.

\section{Results}

During the study period, 6,701 patients aged $70+$ years were admitted, of whom $49.4 \%$ were $70-79$ years, $38.3 \%$ were $80-89$ years and $12.3 \%$ were $90+$ years old.

The 1-year mortality for all age groups combined was $22.4 \% ; 12.7 \%$ died within 3 months, $4.8 \%$ died between 3 and 6 months, $3 \%$ died between 6 and 9 months and $1.9 \%$ died between 9 and 12 months.

1-year mortality rose with age, from $20 \%$ in $70-79$-year-olds to over $25 \%$ in $90+$ year-olds.

\section{Conclusions}

The data from London and Scotland both indicate that a single admission over the age of 70 years old is a significant predictor of death within 1 year.

Authors: ${ }^{1}$ Imperial College and Department of Palliative Care, Imperial College Healthcare NHS Trust, London, UK; ${ }^{2}$ Imperial College Healthcare NHS Trust, London, UK; ${ }^{3}$ Institute of Global Health Innovation, Imperial College, London, UK; ${ }^{4}$ Department of Palliative Care, Imperial College Healthcare NHS Trust, London, UK
An emergency admission over the age of 70 years could be used to aid recognition of the last years of life and to initiate urgent or advance care planning.

The data highlight the need to innovate by sharing electronic, patient-accessible care plans across multiple sites and out-ofhours care providers to improve end-of-life care. Widespread digitalisation could transform practice by bridging gaps in communication, putting patient choice at the centre and reducing unscheduled admissions.

\section{Conflict of interest statement}

None.

\section{Reference}

1 Clark D, Armstrong M, Allan A et al. Imminence of death among hospital inpatients: Prevalent cohort study. Palliat Med 2014;28:4749. 